\title{
The characteristics of intestinal flora in overweight pregnant women and the correlation with gestational diabetes mellitus
}

\author{
Yao Su*, Li Chen*, Dong-Yao Zhang, Xu-Pei Gan, Yan-Nan Cao, De-Cui Cheng, Wen-Yu Liu, Fei-Fei Li, Xian-Ming Xu \\ and Hong-Kun Wang $\mathbb{D}$ \\ Department of Obstetrics and Gynecology, Shanghai General Hospital, Shanghai Jiao Tong University School of Medicine, Shanghai, China
}

Correspondence should be addressed to X-M Xu or H-K Wang: xuxianming77@21cn.com or hongkun_wang821@outlook.com

*(Y Su and L Chen contributed equally to this work)

\begin{abstract}
Objective: To investigate the characteristics of intestinal flora in overweight pregnant women and the correlation with gestational diabetes mellitus (GDM).

Methods: A total of 122 women were enrolled and divided into four groups according to their pre-pregnancy BMI and the presence of GDM: group $1(n=71)$ with a BMI $<24 \mathrm{~kg} / \mathrm{m}^{2}$, without GDM; group $2(n=27)$ with a BMI $<24 \mathrm{~kg} / \mathrm{m}^{2}$, with GDM; group $3(n=17)$ with a $\mathrm{BMI} \geq 24 \mathrm{~kg} / \mathrm{m}^{2}$, without GDM; and group $4(n=7)$ with a BMI $\geq 24 \mathrm{~kg} / \mathrm{m}^{2}$ with GDM. Feces were collected on the day that the oral glucose tolerance test was conducted. The V3-V4 variable region of $16 \mathrm{~S}$ rRNA was sequenced using the Illumina Hiseq 2500 platform, and a bioinformatics analysis was conducted.

Results: There were differences between the four groups in the composition of intestinal flora, and it was significantly different in group 4 than in the other three groups.

Firmicutes accounted for $36.4 \%$ of the intestinal flora in this group, the lowest among the four groups, while Bacteroidetes accounted for $50.1 \%$, the highest among the four groups, making ratio of these two bacteria approximately 3:5, while in the other three groups, this ratio was reversed. In women with a BMI $<24 \mathrm{~kg} / \mathrm{m}^{2}$, the insulin resistance index (homeostatic model assessment for insulin resistance (HOMA-IR)) in pregnant women with GDM was higher than in those without $\left(P_{3}=0.026\right)$.

Conclusion: The composition of the intestinal flora of pregnant women who were overweight or obese before pregnancy and suffered from GDM was significantly different than women who were not overweight or did not suffer from GDM.
\end{abstract}
Key Words
- intestinal flora
- gestational diabetes mellitus
- overweight
- pregnant women
- insulin resistance

\section{Introduction}

Gestational diabetes mellitus (GDM) is one of the most common complications during pregnancy, and the incidence rate is increasing every year and is currently approximately $17.5 \%$ in China (1). GDM is harmful to the long- and short-term health of the mother and the child. However, the etiology of GDM remains unclear. It may be caused by insulin resistance and pancreatic $\beta$-cell secretion defects caused by genetic and environmental factors, but recent studies have revealed that patients who are obese or have type 2 diabetes also have an imbalance in their intestinal flora (2). In particular, the composition of Firmicutes and Bacteroidetes, the two dominant bacteria in the intestinal flora, change in these patients (3). Therefore, the relationship between intestinal flora and GDM has become a focus of recent research. 
This study aims to investigate the characteristics of intestinal flora in pregnant women who were overweight before pregnancy and the correlation between intestinal flora composition and GDM. The aim is to explore the pathogenesis of GDM and provide a basis for the health management of pregnant women who are overweight or obese.

\section{Method}

\section{Subjects}

A total of 122 pregnant women who received antenatal care in the Obstetrics Clinic of the Shanghai First People's Hospital between September 2019 and June 2020 were enrolled in the study.

Inclusion criteria: (1) residents of Shanghai who ate a diet common to the Songjiang District of Shanghai; (2) no diagnosis of diabetes before pregnancy; and (3) normal glucose tolerance in the first trimester of pregnancy.

Exclusion criteria: (1) multigravida; (2) diabetes, hypertension, thyroid disease, gastrointestinal or cardiovascular diseases, or other internal surgical diseases present before pregnancy; (3) the use of assisted reproductive technology; or (4) a history of antibiotic use $\leq 2$ months prior to enrollment.

The study was approved by the ethics committee of the Shanghai General Hospital of the Shanghai Jiao Tong University School of Medicine (No: 2020KY098), and all patients provided signed informed consent (clinical registration number: ChiCTR2000036575). The research flowchart is shown in Fig. 1.

\section{Research methods}

\section{Clinical information collection}

The clinical data of 122 pregnant women were collected from the outpatient electronic medical record system, including height, weight before pregnancy, age, number of pregnancies and deliveries, and blood pressure.

The most common maternal characteristics observed were fasting plasma glucose levels (FPG), 1-h plasma glucose, 2-h plasma glucose (2hPG), fasting insulin (FINS), 1-h plasma insulin (1hPIN), 2-h plasma insulin (2hPIN), homeostatic model assessment for insulin resistance (HOMA-IR), triglyceride (TG), total cholesterol, HDL, and LDL, which were obtained from the patients' medical records. On the day the blood was drawn, the serum aliquots were collected and stored at $-80^{\circ} \mathrm{C}$.

\section{Criteria and grouping}

According to the recommended standard of the Chinese Guidelines for the Prevention and Control of Overweight and Obesity in Adults, a BMI $\geq 24.0 \mathrm{~kg} / \mathrm{m}^{2}$ but $<28.0 \mathrm{~kg} / \mathrm{m}^{2}$ indicates that the patient is overweight, while a $\mathrm{BMI} \geq 28.0 \mathrm{~kg} / \mathrm{m}^{2}$ indicates that they are obese (4). Prepregnancy BMI = weight $(\mathrm{kg}) /$ height $\left(\mathrm{m}^{2}\right)$.

A diagnosis of GDM can be confirmed using a $75 \mathrm{~g}$ oral glucose tolerance test (OGTT), with blood glucose levels

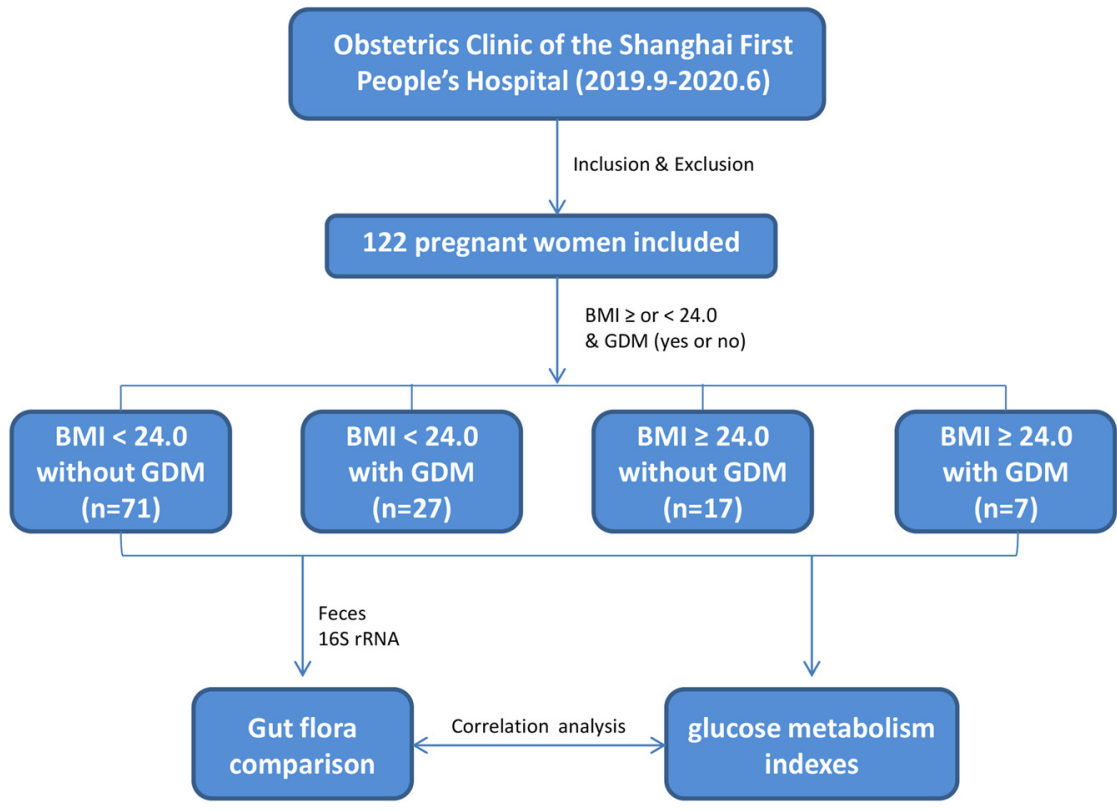

Figure 1

Research flowchart.

https://ec.bioscientifica.com https://doi.org/10.1530/EC-21-0433 (c) 2021 The authors Published by Bioscientifica Ltd

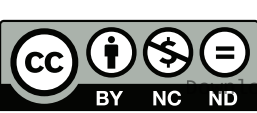

This work is licensed under a Creative Commons Attribution-NonCommercial-NoDerivatives 4.0 elfternationad ficense ifica . com at 04/26/2023 10:05:28Am 
when fasting and 1 and $2 \mathrm{~h}$ after ingesting the glucose of $\geq 5.1, \geq 10.0$, or $\geq 8.5 \mathrm{mmol} / \mathrm{L}$, respectively (5).

The subjects were divided into four groups according to their pre-pregnancy BMI and the presence of GDM: group $1(n=71)$ with a BMI $<24 \mathrm{~kg} / \mathrm{m}^{2}$, without GDM; group 2 $(n=27)$ with a BMI $<24 \mathrm{~kg} / \mathrm{m} 2$, with GDM; group $3(n=17)$ with a BMI $\geq 24 \mathrm{~kg} / \mathrm{m}^{2}$, without GDM; and group $4(n=7)$ with a BMI $\geq 24 \mathrm{~kg} / \mathrm{m}^{2}$, with GDM.

\section{Homeostasis model assessment insulin resistance}

HOMA-IR = fasting plasma glucose $(\mathrm{mmol} / \mathrm{L}) \times$ FINS

$(\mathrm{U} / \mathrm{mL}) / 22.5$.

\section{Collection of fecal samples and the extraction, amplification, database construction, and bioinformatics analysis of fecal DNA}

On the same day as the OGTT was conducted, 2-3 g (or four scoops) of fresh feces were collected with a sampler, soaked in a preservation solution, and stirred with a sampling spoon to create a uniform mixture. The mixture was then stored at $-80^{\circ} \mathrm{C}$ by a specially assigned person after admission.

Total bacterial DNA was extracted from the fecal samples using a magnetic fecal DNA extraction kit, and the quality of the extracted DNA was detected using a Qubit dsDNA HS Assay Kit (Carlsbad, CA, USA). After eliminating the samples that did not meet the test standard, the V3-V4 variable region of the 16S rRNA was specifically amplified by PCR. PCR products were detected using 2\% agarose gel electrophoresis and sequenced using the Illumina Hiseq2500 system, after which a gene library was established. The Ribosomal Database Project classifier Bayesian algorithm was used in the Trimmomatic software to classify the operational taxonomic unit (OTU) representative sequences with a 97\% similarity level. The community composition of the samples was analyzed at all levels (kingdom, phylum, class, order, family, genus, and species) and clustered into OTUs before being compared with data in the SILVA rRNA database. The species classification information was annotated. Using the USEARCH software platform, the samples were subjected to bioinformatics analysis, including relative species abundance, OTUs (species richness), and diversity (Ace, Chao, Shannon Diversity, and Simpson's Diversity index). Pattern recognition analysis based on a forward feature selection combined with linear discriminant analysis was performed using R version 3.5.1.

\section{Statistical analysis}

The data were analyzed using the SPSS 22.0 statistical software. Normally distributed measurement data were expressed as mean \pm S.D. $(x \pm$ S.D. $)$, and the data of different groups were compared using an independent sample $t$-test. Non-normally distributed measurement data were expressed as mean and interquartile range and were compared using a Mann-Whitney $U$ or $\chi^{2}$ test. $P<0.05 / 4=0.0083$ was considered statistically significant. Spearman's rank correlation test was used for correlation analysis between different groups at the phylum or genus levels.

\section{Results \\ Clinical data}

There were no significant differences in age, gestational age, gravidity, birth order, or systolic and diastolic blood pressure between the four groups (see Table 1).

\section{Changes in intestinal flora composition}

The comparison of the relative abundance of phylum and genus levels of intestinal flora between the four groups

At the phylum level, 22 phyla were found in the intestinal flora of the 122 subjects. The five most common phyla of average abundance were Firmicutes, Bacteroidetes, Proteobacteria, Actinobacteria, and Verrucomicrobia. The composition ratio of intestinal flora at the phylum level in the four groups is shown in Fig. 2A. At the genus level, 315 genera were found in the intestinal flora of the subjects. The eight most common genera of average abundance were Bacteroides, Faecalibacterium, Megamonas, Ruminococceae Ucg002, Bifidobacterium, Other_A, Lachnoclostridium, and Escherichia-Shigella. The composition ratio of intestinal flora at the genus level in the four groups is shown in Fig. 2B.

As shown in Fig. 3, when compared with groups 1, 2, and 3, in group 4, Firmicutes accounted for 36\%, which was significantly lower than in the other groups $(54,57$, and $56 \%$, respectively); Bacteroidetes accounted for about $50.1 \%$, which was significantly higher than in the other groups (31, 30, and 31\%, respectively); and Bacteroides accounted for $48 \%$, which was also significantly higher than in the other three groups $(24,25$, and $25 \%$, respectively). The percentage of Megamonas in the patients in group 3 was higher than in the other three groups. 
Table 1 The characteristics of 122 pregnant women.

\begin{tabular}{|c|c|c|c|}
\hline & & Pre-BMI & $4 \mathrm{~kg} / \mathrm{n}$ \\
\hline & & Non-GDM $(n=71)$ & GD \\
\hline Age & $(\bar{x} \pm$ S.D., years) & $30.6 \pm 4.47$ & 30 \\
\hline Ges & ational age (M(P25-P75) (weeks) & $25.55(25-26)$ & 25. \\
\hline Gra & idity (M(P25-P75)) & $2.14(1-3)$ & 2. \\
\hline Pari & $y(M(P 25-P 75))$ & $0.45(0-1)$ & 0. \\
\hline syst & olic pressure ( $\bar{x} \pm$ S.D., mmhg) & $106.61 \pm 9.41$ & 112. \\
\hline dias & tolic pressure ( $\bar{x} \pm$ S.D., mmhg) & $69.20 \pm 5.84$ & 70. \\
\hline & $\begin{array}{l}\text { Iaracteristics, biochemical and } \mathrm{r} \\
\text { E.M. when normally distributed }\end{array}$ & $\begin{array}{l}\text { al variables of } \\
\text { an with } 25-75\end{array}$ & $\begin{array}{l}\text { hd no } \\
\text { quart }\end{array}$ \\
\hline $\begin{array}{l}\text { The } \\
\text { bet }\end{array}$ & $\begin{array}{l}\text { comparison of the composit } \\
\text { ween the four groups }\end{array}$ & of intestin & lora \\
\hline (1) & $\begin{array}{l}\text { Groups } 1 \text { and } 2 \text { : There was no } \\
\text { the composition of intestinal } \\
\text { women with and without GD }\end{array}$ & $\begin{array}{l}\text { gnificant differe } \\
\text { ora between pre } \\
\text { I. }\end{array}$ & $\begin{array}{l}\text { ce in } \\
\text { nant }\end{array}$ \\
\hline (2) & $\begin{array}{l}\text { Groups } 3 \text { and } 4: \text { The } \\
\text { Firmicutes was significantly } \\
\text { group } 3\left(P_{2}=0.017\right) \text {, and the } \\
\text { Bacteroidetes and Bacteroides } \\
\left(P_{2}=0.027 \text { and } 0.02 \text {, respective }\right.\end{array}$ & $\begin{array}{l}\text { ative abundar } \\
\text { wer in group } \\
\text { elative abunda } \\
\text { as significantly } \\
\text { y. }\end{array}$ & $\begin{array}{l}\text { of } \\
\text { than } \\
\text { gher of } \\
\text { gher }\end{array}$ \\
\hline
\end{tabular}

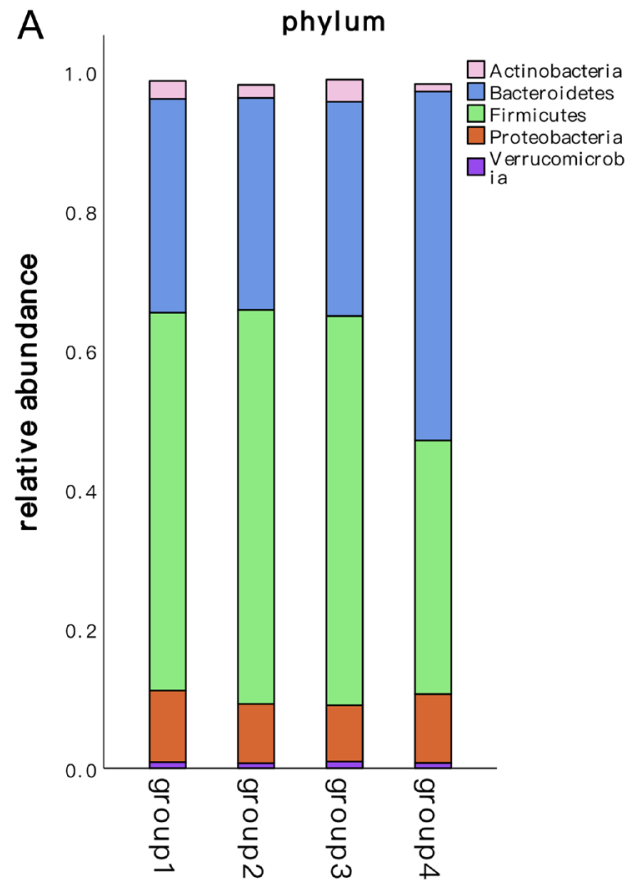

(3) Groups 1 and 3: There was no difference in the composition of intestinal flora at the phylum level between group 1 and 3. However, the relative abundance of Megamonas was significantly higher in group 3 than group $1\left(P_{3}=0.025\right)$.

(4) Groups 2 and 4: The relative abundance of intestinal Firmicutes and Ruminococceae_Ucg002 was lower, and the relative abundance of Bacteroidetes and Bacteroides was higher in group 4 than in group 2 $\left(P_{4}=0.015,0.039,0.022\right.$, and 0.016 , respectively; see Table 2).

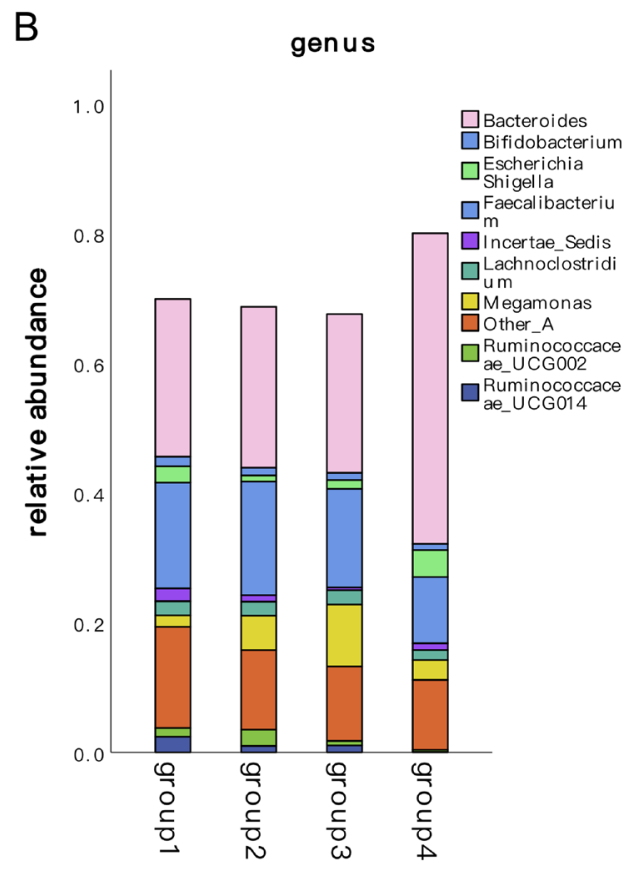

Figure 2

Histogram of the distribution of intestinal flora in 122 pregnant women at the phylum and genus levels.

https://ec.bioscientifica.com https://doi.org/10.1530/EC-21-0433 (c) 2021 The authors Published by Bioscientifica Ltd

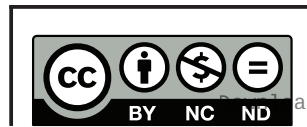

This work is licensed under a Creative Commons Attribution-NonCommercial-NoDerivatives 4.0 Internationab ficense.ifica . com at $04 / 26 / 2023 \quad 10: 05: 28 \mathrm{Am}$ 
A

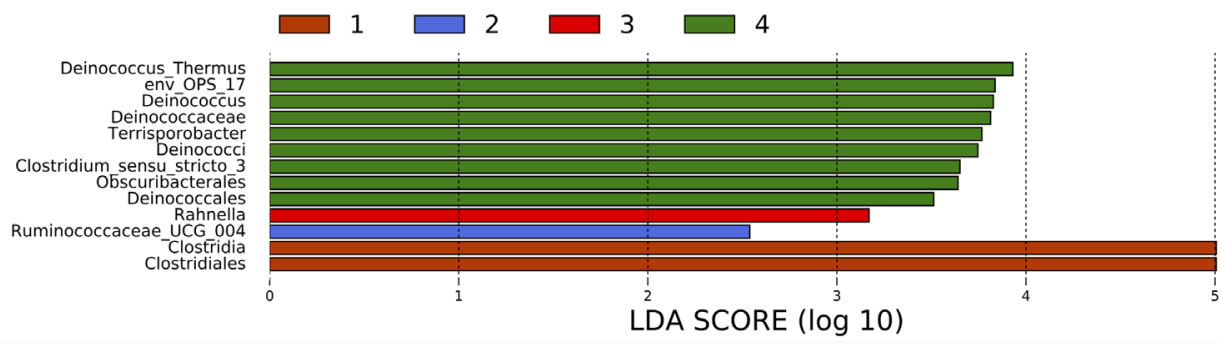

B
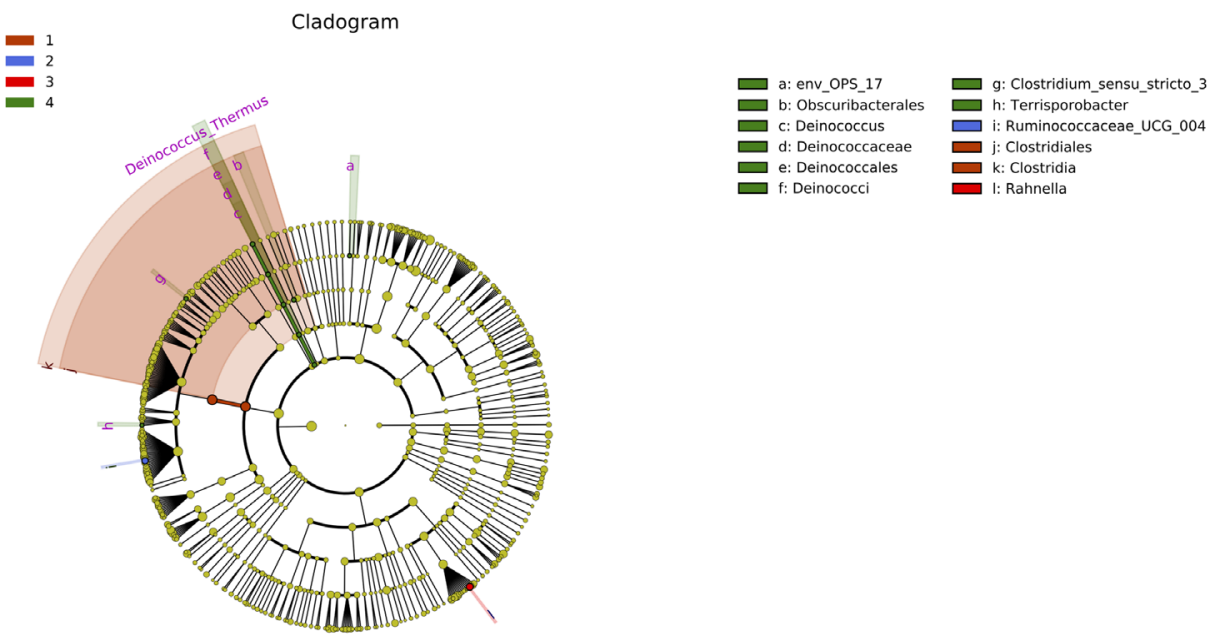
f: Deinococcales

\section{Figure 3}

Cladistic maps of biomarkers for the genus-level classification by linear discriminant analysis effect size. Different colors indicate the florae that were rich in different groups: the scarlet circle indicates group 1, the blue circle indicates group 2, the red circle indicates group 3, the green circle indicates group 4 , and the yellow circle indicates the common genus of the four groups.

\section{The comparison of the diversity and OTUs of intestinal flora}

It was found that, no matter what a patient's BMI and blood glucose level were before pregnancy, the abundance and diversity (Ace index, Chao index, Shannon index, and Simpson index) of the intestinal flora in the four groups were similar, and none of the differences between the groups were statistically significant. Similarly, there was no significant difference between the four groups in the total number of OTUs (see Table 3).

\section{Comparison of insulin level and HOMA-IR between the four groups}

The HOMA-IR was significantly higher in group 3 than in group $1\left(P_{2}=0.001\right)$ and was also higher in patients with GDM than those without $\left(P_{3}=0.026\right)$. However, there was no significant difference in HOMA-IR between group 3 and 4 or between group 2 and 4 (see Table 4).

\section{Identification of the biomarkers of intestinal flora at the genus level in the four groups}

Linear discriminant analysis effect size was used to identify the biomarkers of intestinal flora at the genus level. The results revealed that the four groups had different marker florae, as shown in Fig. 3. The marker florae in group 1 were Clostridia and Clostridiales, and the marker flora in group 2 was Ruminococcaceae_UCGO14 and in group 3 was Rahnella. In group 4, there were nine marker florae, and Deinococcus was the characteristic marker from the phylum level to the genus level, but the markers in group 4 also included Obscuribacteralesjun, Clostridium_sensu_stricto_3, and Terrisporobacter.

\section{The correlation between the relative abundance of flora and the biochemical metabolic indexes of the four groups}

The correlation analysis of the dominant intestinal bacteria and the glucose metabolism indexes of the women in all https://ec.bioscientifica.com https://doi.org/10.1530/EC-21-0433 (c) 2021 The authors Published by Bioscientifica Ltd
This work is licensed under a Creative Commons Attribution-NonCommercial-NoDerivatives 4.0 elnternationab dicense ifica.com at 04/26/2023 10:05:28AM 
Кำ ปิ๊ ó 00 \| 0 || || ||

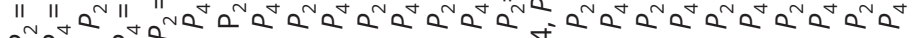
कर

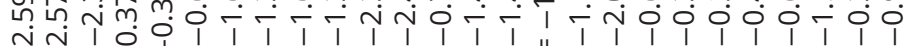
|

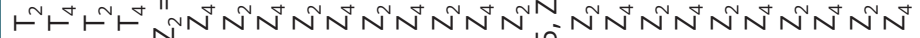

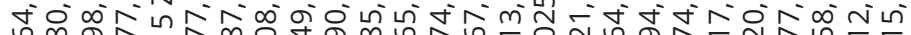

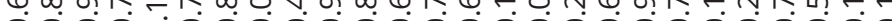

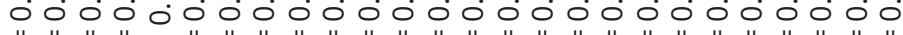

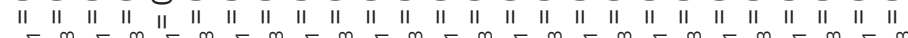
$a^{-} a^{m} a^{m} a^{m} a^{m} a^{m} a^{m} a^{m} a^{m} a^{m} a^{m} a^{m} a^{m} a^{m} a^{m} a^{m} a^{-} a^{m} a^{m}$

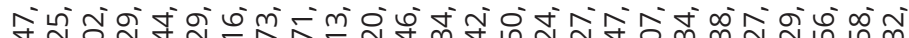

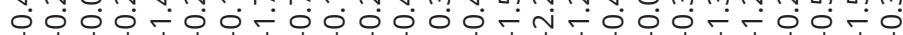
" NMNN

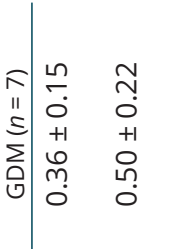

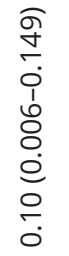

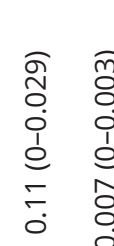

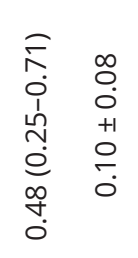

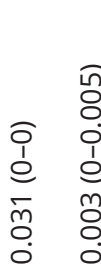

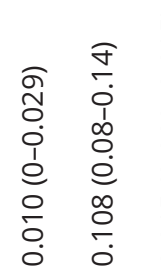

In
o
0
1
$n$
0
0
0
ñ
0
0
0

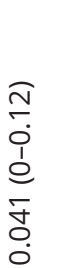

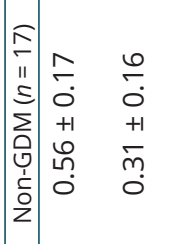

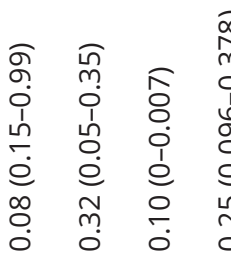

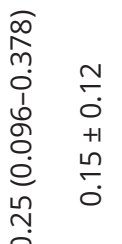

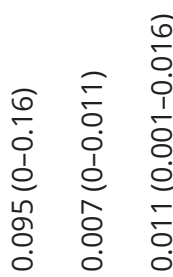

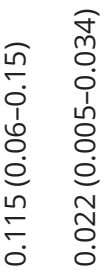

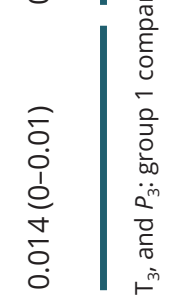

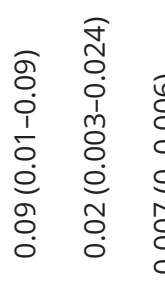

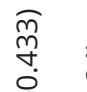

๙

$\stackrel{2}{s}$ in

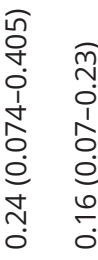

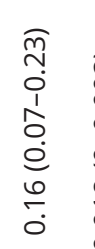
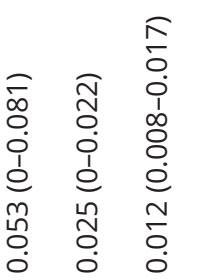

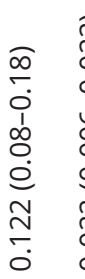

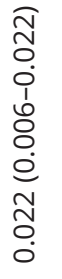

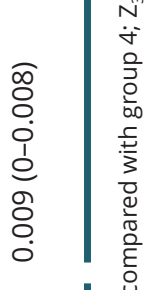

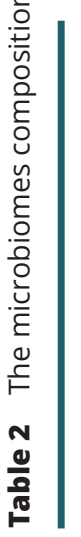

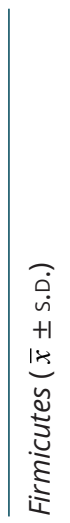

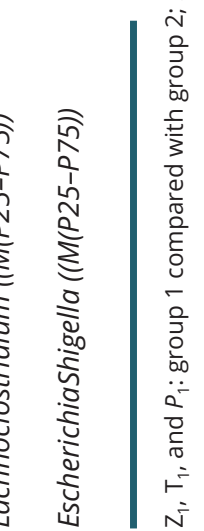




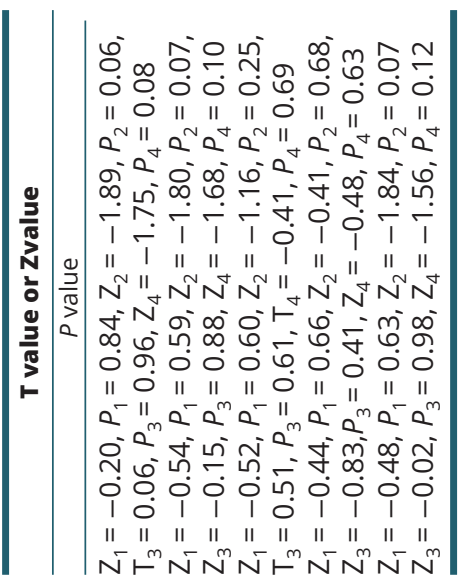

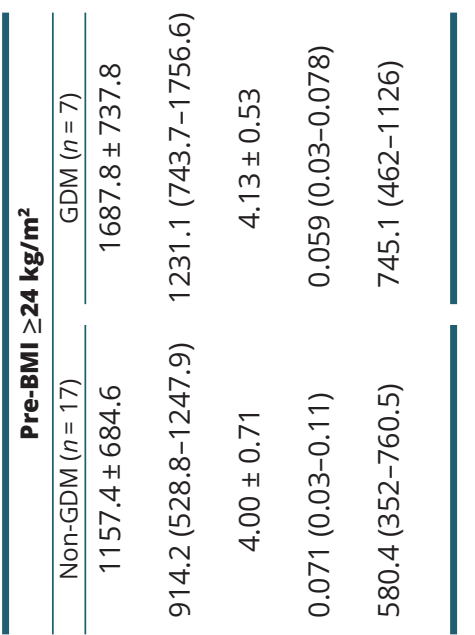

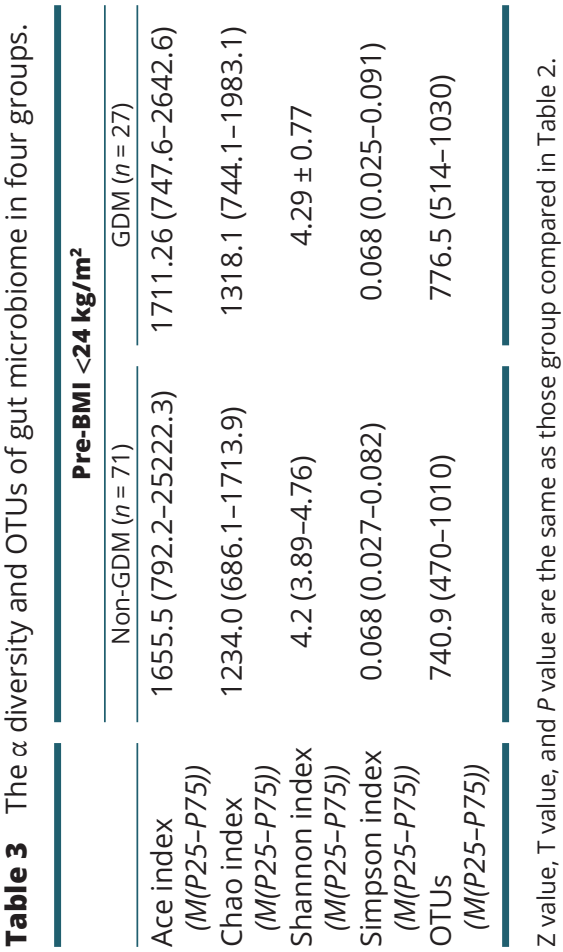

https://ec.bioscientifica.com https://doi.org/10.1530/EC-21-0433 (c) 2021 The authors Published by Bioscientifica Ltd four groups is shown in Fig. 3, with the correlation at the phylum level shown in Fig. 4A and the genus level shown in Fig. 4B.

In group 4, the relative abundance of Firmicutes was negatively correlated and Bacteroidetes was positively correlated with $1 \mathrm{hPIN}$ levels, and the relative abundance of Actinomycetes was positively correlated with $2 \mathrm{hPIN}$ levels. The relative abundance of Verrucomicrobia was positively correlated with HDL, and Tenericutes was negatively correlated with glycated hemoglobin (HbA1C) levels.

The relative abundance of Bacteroides and Faecalis was positively correlated with FINS and HDL, respectively. The relative abundance of Ruminococcaceae_UCG014 was negatively correlated with Hb1AC, FPG, FINS, 1hPIN, 2hPIN, HOMA-IR, and TG. The relative abundance of Incertae-Sedis was negatively correlated with FPGs, and the relative abundance of Eubacterium_coprostanoligenes_ group, Ruminococcaceae_UCGOO2, and Christensenellaceae $R 7$ group were positively correlated with $2 \mathrm{hPG}$. The relative abundance of Ruminococcaceae_UCG002 was negatively correlated with FINS and 1hPIN levels, and Christensenellaceae_R7_group and Ruminoccus_1 was positively correlated with HDL. However, the relative abundance of Hafnia was negatively correlated with HDL.

\section{Discussion}

Intestinal florae have rich functions and are symbiotic with the host. They not only participate in the routine digestive process by assisting with the digestion and absorption of nutrients but also maintain the intestinal biological barrier by antagonizing the colonization of and infection by pathogenic microorganisms. They can also stimulate and regulate the cellular and humoral immunity of the human body. There are many species of intestinal florae, but they are mainly composed of Firmicutes, Bacteroidetes, Proteus, Actinomycetes, Fusobacteria, and Verrucomicrobia. The first four of these account for $98 \%$ of the total intestinal flora, and Firmicutes and Bacteroidetes account for 64 and 23\%, respectively (1). They are therefore the main components of intestinal flora.

Obesity, a sedentary lifestyle, eating habits, and the use of antibiotics can all lead to an imbalance in intestinal flora (6). When the balance of Firmicutes and Bacteroidetes is changed, obesity and diabetes may occur. In the intestinal flora of obese mice, Firmicutes has been found to decrease by $50 \%$, while Bacteroidetes increases by $50 \%$ (3). A study of obese children revealed that this phenomenon also exists in humans, with the percentage of Firmicutes in their intestinal flora increasing and Bacteroidetes decreasing (7).

This work is licensed under a Creative Commons Attribution-NonCommercial-NoDerivatives 4.0 Internationad ticense.ifica. com at $04 / 26 / 2023$ 10:05:28AM 
Table 4 The comparison of HOMA-IR in four groups.

\begin{tabular}{|c|c|c|c|c|c|}
\hline & \multicolumn{2}{|c|}{ Pre-BMI $<\mathbf{2 4} \mathbf{~ k g} / \mathbf{m}^{\mathbf{2}}(n=98)$} & \multicolumn{2}{|c|}{ Pre-BMI $\geq 24 \mathbf{~ k g} / \mathbf{m}^{2}(n=24)$} & \multirow{2}{*}{$\begin{array}{c}\text { T value or } \mathbf{Z} \text { value } \\
P \text { value }\end{array}$} \\
\hline & Non-GDM $(n=71)$ & $\operatorname{GDM}(n=27)$ & Non-GDM $(n=17)$ & $\operatorname{GDM}(n=7)$ & \\
\hline HOMA-IR (M(P25-P75)) & $1.30(0.89-1.71)$ & $1.71(1.15-2.25)$ & $2.09(1.27-3.10)$ & $2.25(1.58-2.27)$ & $\begin{array}{l}\mathrm{Z}_{0}=-3.78, P_{0}=0 \mathrm{Z}_{1}=-2.23, P_{1}=0.026 \\
\mathrm{Z}_{2}=-0.83, P_{2}=0.41 \mathrm{Z}_{3}=-3.42, P_{3}=0.001 \\
\mathrm{Z}_{4}=-1.66, P_{4}=0.097\end{array}$ \\
\hline
\end{tabular}

$\mathrm{Z}_{0}, P_{0}$ : the group have pre- $\mathrm{BMI}<24$ compared with pre- $\mathrm{BMI} \geq 24$. $\mathrm{Z}$ value, $\mathrm{T}$ value, and $P$ value are the same as those group compared in $\mathrm{Table} 2$.

The present study found that Firmicutes and Bacteroidetes were the two dominant florae in the intestinal flora of pregnant women. However, in pregnant women with GDM who were overweight or obese, the relative abundance of Bacteroidetes was significantly higher and the relative abundance of Firmicutes was lower than in women who were not overweight and women who were overweight but did not suffer from GDM. Firmicutes accounted for $36.4 \%$ of the intestinal flora in group 4 , which was the lowest of the four groups, while Bacteroidetes accounted for $50.1 \%$, which was the highest of the four groups. The ratio of the two bacteria in this group was approximately 3:5, but in the other three groups, this ratio was reversed.

A previous study on the structural changes of intestinal flora in people with type 2 diabetes found that the relative abundance of Firmicutes decreased and of Bacteroidetes and Proteus increased significantly (8). In addition, the percentage of Bacteroidetes and Firmicutes has been found to be significantly positively correlated with a decrease in glucose tolerance (9). Bacteroidetes has approximately 20 genera, of which Bacteroides is the most abundant in the human gastrointestinal tract (10). A previous study involving 345 patients with type 2 diabetes found that high levels of Bacteroidetes were positively correlated with the occurrence of diabetes (11).

The possible mechanisms of intestinal flora imbalance leading to obesity and diabetes are: (1) changes to Firmicutes and Bacteroidetes in the intestine can lead to an increase in the total heat absorbed from food, thus decreasing the energy lost in stools $(12,13,14)$; (2) the interaction of intestinal flora with environmental and genetic factors in patients with diabetes can lead to an increase in intestinal permeability and a change in mucosal immune response, thereby leading to the progression or deterioration of diabetes (15); and (3) the intestinal microbiota are also responsible for energy production through the anaerobic decomposition of dietary fiber, protein, and peptides to produce short-chain fatty acids (SCFAs). Escherichia coli produces the most acetate, propionate, and butyrate (16). Acetate and propionate are primarily produced by Bacteroidetes, and butyrate by Firmicutes. Cao et al. (17) believed that SCFAs, especially butyrate, can reduce the inflammatory response of adipocytes and improve insulin sensitivity and secretion by stimulating the secretion of peptide 1 , such as glucagon-1 $(18,19,20)$. When the number of Firmicutes producing butyrate decreases, the beneficial effect is greatly weakened.

Obesity is associated with insulin resistance. The present study found that patients in group 3 and 4 had higher HOMA-IR levels. There was no difference in insulin resistance, but there was a significant difference in the composition of the intestinal flora between these two groups. In groups 1 and 2, the basic levels of HOMA-IR were low, and the level of insulin resistance in group 2 was significantly higher than in group 1 . However, there was no significant difference in the composition of intestinal flora between these two groups. Therefore, in pregnant women with a normal pre-pregnancy weight, insulin resistance may be an important cause of the increase in blood glucose, but for pregnant women who are overweight or obese before pregnancy, the imbalance of intestinal flora and metabolic changes may be an important mechanism affecting the occurrence of GDM.

These results indicate that for pregnant women with BMI $\geq 24 \mathrm{~kg} / \mathrm{m}^{2}$, the regulation of intestinal flora may effectively improve abnormal glucose metabolism and prevent the occurrence of GDM. One intervention choice for metabolic diseases is probiotics, which can safely and effectively alter the intestinal flora. For example, some species of Bifidobacteria and Lactobacillus have significant benefits in regulating the intestinal microbiota, as they can create a beneficial intestinal environment and help maintain a healthy immune system $(21,22)$. Previous studies have found that improvement in the symptoms of type 2 diabetes, such as improved intestinal integrity, reduced systemic lipopolysaccharide levels, reduced endoplasmic reticulum stress, and improved peripheral insulin sensitivity, can be observed after intervention with probiotics $(23,24,25)$. In addition, changing the dietary pattern can help regulate the intestinal flora, for example, avoiding a high-fat diet can improve the diversity of intestinal microflora (26), while a highcarbohydrate diet can increase the abundance of intestinal flora (13) and the levels of Bifidobacterium (27).

This work is licensed under a Creative Commons Attribution-NonCommercial-NoDerivatives 4.0 elnternational License.ifica, com at $04 / 26 / 2023$ 10:05:28AM 
A

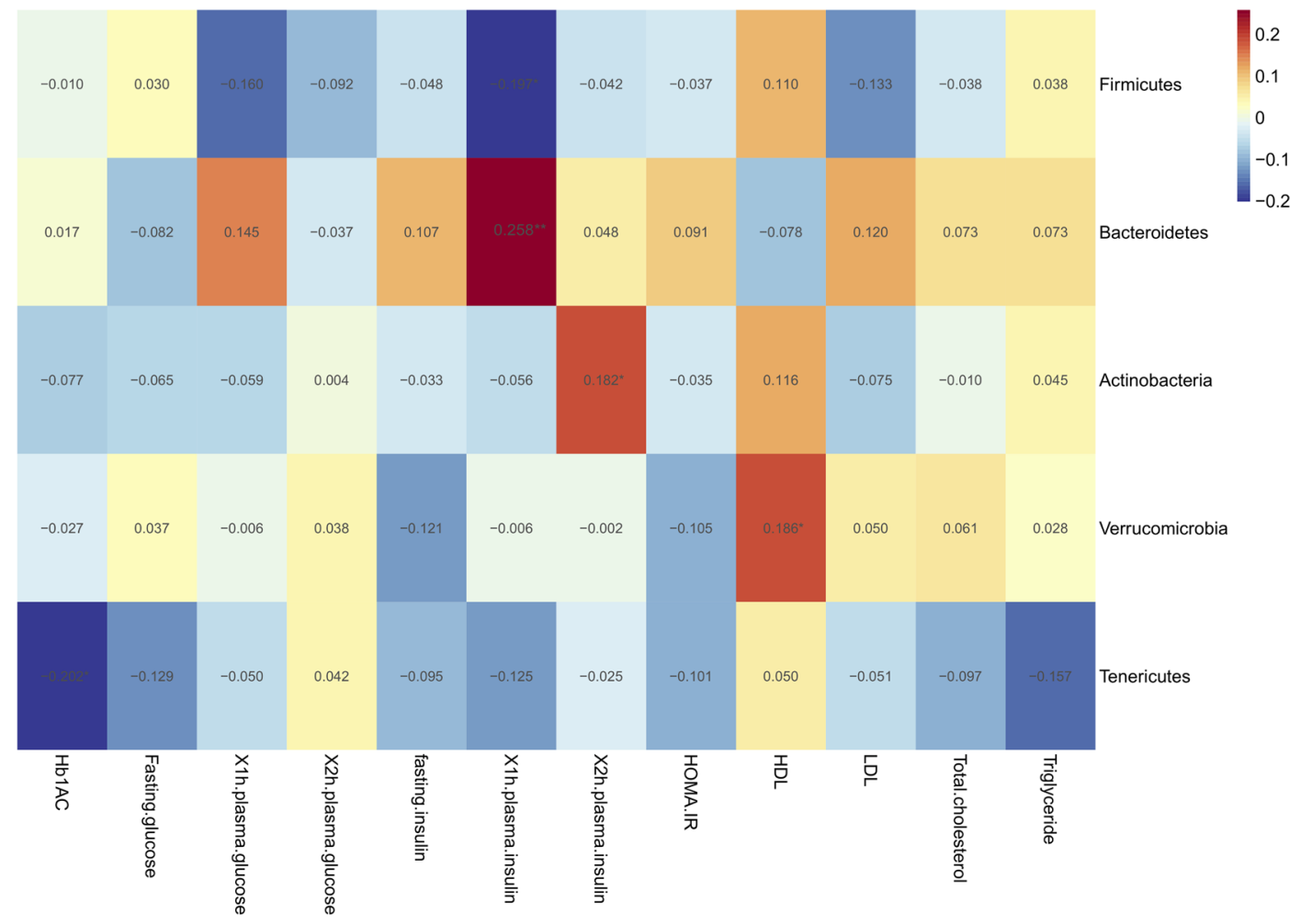

B

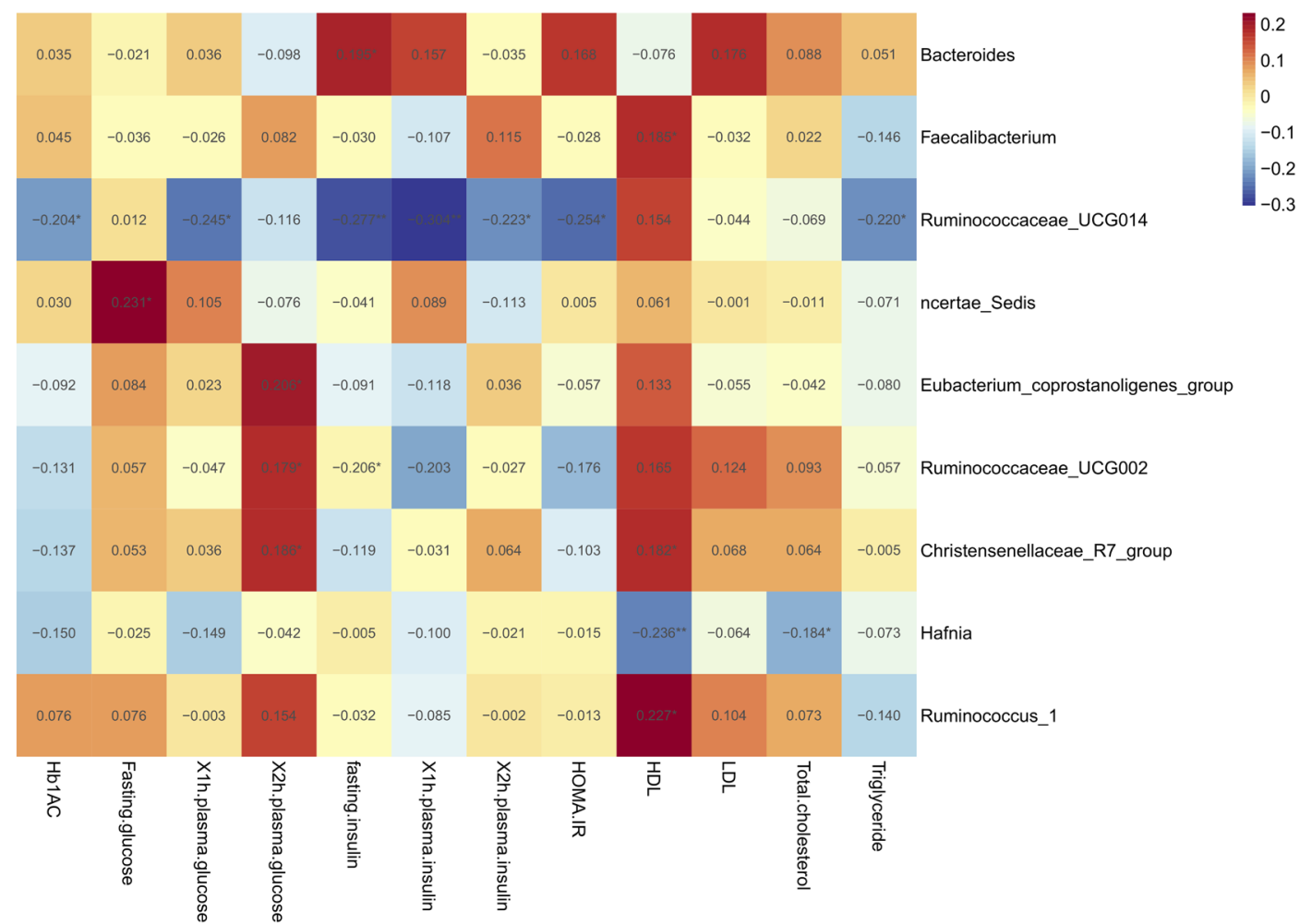

\section{Figure 4}

Correlation between the intestinal flora at the phylum and genus levels and the blood glucose metabolism indexes of 122 pregnant women. Correlation analysis of the clinical blood glucose metabolism indexes and the characteristic bacteria. In Spearman correlation analysis, $* P<0.05$ and $* *$ indicates $P<0.01$. 
In addition, fecal microbiota transplantation (FMT), a method of transplanting healthy individual flora into a patient's colon to reconstitute the intestinal flora, can be used, and 6 weeks of FMT treatment in patients with metabolic syndrome has been found to improve insulin sensitivity and increase the number of butyrateproducing intestinal microorganisms (28).

In summary, this study focuses on the characteristics of the flora composition in pregnant women with different pre-pregnancy BMIs when they are diagnosed with GDM. In women with normal blood glucose levels, irrespective of the BMI, the composition of the intestinal flora is similar. The converse is also true, that is, in women with a normal BMI, regardless of the blood glucose level, the composition of the flora is also similar. However, in pregnant women who are overweight and have GDM, the flora composition is significantly different from those with a normal BMI and GDM. The HOMA-IR of pregnant women in group 2 was significantly higher than in group 1 and 3 , and this implies that the HOMA-IR of women in group 4 is similarly higher than in group 1 and 3 . In addition to the effect of insulin resistance, an unbalanced intestinal flora composition in pregnant women, especially the ratio of Firmicutes to Bacteroides, influences glucose metabolism, raising blood sugar. GDM in pregnant women with a normal BMI is primarily caused by insulin resistance.

However, there are some deficiencies in this study. First, since our hospital did not perform OGTT tests on all patients before their pregnancy, women with prior impaired glucose tolerance may be included. This is a huge limitation to the results, and the research results need to be verified by more rigorous experiments in the future. Second, the study only covered the period before the second trimester, rather than the whole pregnancy, so it was not possible to know the dynamic composition of the intestinal flora of pregnant women throughout their pregnancy. Follow-up studies should extend this study to include the composition of microflora in the first trimester, the third trimester, and the postpartum period. Third, the patients included in this study were all from Shanghai, and the food culture in Shanghai is unique, which may lead to differences in the composition of intestinal flora when compared with other regions. Further research is needed to verify whether the results are applicable to other regions or countries.

\section{Conclusion}

The present study found that pregnant women with GDM who were overweight or obese prior to pregnancy had an obvious imbalance in intestinal flora. In particular, the relative abundance of Bacteroidetes was significantly increased, while the relative abundance of Firmicutes was significantly decreased when compared with women with a normal pre-pregnancy BMI. This may be an important mechanism for GDM in pregnant women with high prepregnancy BMIs. Therefore, clinical practice should offer supplementary probiotics as an intervention and focus on improving the dietary habits of pregnant women who are overweight or obese. FMT treatment can also be carried out, if necessary, thereby reducing the risk of GDM.

\section{Declaration of interest}

The authors declare that there is no conflict of interest that could be perceived as prejudicing the impartiality of the research reported.

\section{Funding}

Three year action plan for promoting clinical skills and clinical innovation ability of municipal hospitals (No. SHDC2020CR2060B). The funding body had no role in the design of the study and collection, analysis, and interpretation of data and in writing the manuscript.

\section{Ethics approval and consent to participate}

This study was conducted with approval from the Ethics Committee of Shanghai General Hospital, Shanghai Jiao Tong University school of Medicine (No. 2020KY098). Written informed consent was obtained from all patients.

\section{Consent for publication}

All participants signed a document of informed consent.

\section{Availability of data and materials}

The datasets used and/or analyzed during the current study available from the corresponding author on reasonable request.

\section{Author contribution statement}

$D Y Z, X P G, Y N C$, and D C C conceived the idea and conceptualized the study. F F L, X M X, and $W Y L$ collected the data. $W Y L, F F L$, and $X M X$ analyzed the data. $Y S, L C$, and $\mathrm{H} \mathrm{K} \mathrm{W}$ drafted the manuscript, then $Y \mathrm{~S}$ and $\mathrm{H} \mathrm{K} \mathrm{W}$ reviewed the manuscript. All authors read and approved the final draft.

\section{Acknowledgements}

The authors would like to acknowledge the hard and dedicated work of all the staff that implemented the intervention and evaluation components of the study.

\section{References}

1 Tremaroli V \& Bäckhed F. Functional interactions between the gut microbiota and host metabolism. Nature 2012489 242-249. (https:// doi.org/10.1038/nature11552) 
2 Salah M, Azab M, Ramadan A \& Hanora A. New insights on obesity and diabetes from gut microbiome alterations in Egyptian adults. Omics 201923 477-485. (https://doi.org/10.1089/omi.2019.0063)

3 Al-Attas OS, Al-Daghri NM, Al-Rubeaan K, da Silva NF, Sabico SL, Kumar S, McTernan PG \& Harte AL. Changes in endotoxin levels in T2DM subjects on anti-diabetic therapies. Cardiovascular Diabetology 20098 20. (https://doi.org/10.1186/1475-2840-8-20)

4 Wang YF, Sun XM, Xue H, Zhao WH, Yang XG, ZHu XY, Zhao L \& Yang YX. Interpretation of China's blue book on obesity prevention and control in China and suggestions on obesity prevention and control measures in China. Zhong Hua Yu Fang Yi Xue Za Zhi 201953 875-884. (https://doi.org/10.3760/cma.j.issn.0253?9624.2019.09.003)

5 Olabi B \& Bhopal R. Diagnosis of diabetes using the oral glucose tolerance test. BMJ 2009339 b4354. (https://doi.org/10.1136/bmj. b4354)

6 Jandhyala SM, Talukdar R, Subramanyam C, Vuyyuru H, Sasikala M \& Nageshwar Reddy D. Role of the normal gut microbiota. World Journal of Gastroenterology 201521 8787-8803. (https://doi.org/10.3748/wjg. v21.i29.8787)

7 Indiani CMDSP, Rizzardi KF, Castelo PM, Ferraz LFC, Darrieux M \& Parisotto TM. Childhood obesity and firmicutes/bacteroidetes ratio in the gut microbiota: a systematic review. Childhood Obesity 201814 501-509. (https://doi.org/10.1089/chi.2018.0040)

8 Roager HM, Vogt JK, Kristensen M, Hansen LBS, Ibrügger S, Mærkedahl RB, Bahl MI, Lind MV, Nielsen RL, Frøkiær H, et al. Whole grain-rich diet reduces body weight and systemic lowgrade inflammation without inducing major changes of the gut microbiome: a randomised cross-over trial. Gut 201968 83-93. (https://doi.org/10.1136/gutjnl-2017-314786)

9 Larsen N, Vogensen FK, van den Berg FW, Nielsen DS, Andreasen AS, Pedersen BK, Al-Soud WA, Sørensen SJ, Hansen LH \& Jakobsen M. Gut microbiota in human adults with type 2 diabetes differs from non-diabetic adults. PLOS ONE 20105 e9085. (https://doi.org/10.1371/ journal.pone.0009085)

10 Ley RE, Bäckhed F, Turnbaugh P, Lozupone CA, Knight RD \& Gordon JI. Obesity alters gut microbial ecology. PNAS 2005102 11070-11075. (https://doi.org/10.1073/pnas.0504978102)

11 Qin J, Li Y, Cai Z, Li S, Zhu J, Zhang F, Liang S, Zhang W, Guan Y, Shen D, et al. A metagenome-wide association study of gut microbiota in type 2 diabetes. Nature 2012490 55-60. (https://doi.org/10.1038/ nature11450)

12 Clemente JC, Ursell LK, Parfrey LW \& Knight R. The impact of the gut microbiota on human health: an integrative view. Cell 2012148 1258-1270. (https://doi.org/10.1016/j.cell.2012.01.035)

13 Koliada A, Syzenko G, Moseiko V, Budovska L, Puchkov K, Perederiy V, Gavalko Y, Dorofeyev A, Romanenko M, Tkach S, et al. Association between body mass index and firmicutes/bacteroidetes ratio in an adult Ukrainian population. BMC Microbiology 201717 120. (https:// doi.org/10.1186/s12866-017-1027-1)

14 Jumpertz R, Le DS, Turnbaugh PJ, Trinidad C, Bogardus C, Gordon JI $\&$ Krakoff J. Energy-balance studies reveal associations between gut microbes, caloric load, and nutrient absorption in humans. American Journal of Clinical Nutrition 201194 58-65. (https://doi.org/10.3945/ ajcn.110.010132)

15 Razmpoosh E, Javadi A, Ejtahed HS, Mirmiran P, Javadi M \& Yousefinejad A. The effect of probiotic supplementation on glycemic control and lipid profile in patients with type 2 diabetes: a randomized placebo controlled trial. Diabetes and Metabolic Syndrome 201913 175-182. (https://doi.org/10.1016/j.dsx.2018.08.008)
16 Baxter NT, Lesniak NA, Sinani H, Schloss PD \& Koropatkin NM. The glucoamylase inhibitor acarbose has a diet-dependent and reversible effect on the murine gut microbiome. mSphere 20194 e00528-18. (https://doi.org/10.1128/mSphere.00528-18)

17 Gao Z, Yin J, Zhang J, Ward RE, Martin RJ, Lefevre M, Cefalu WT \& Ye J. Butyrate improves insulin sensitivity and increases energy expenditure in mice. Diabetes 200958 1509-1517. (https://doi.org/10.2337/db08-1637)

18 Ríos-Covián D, Ruas-Madiedo P, Margolles A, Gueimonde M, de Los Reyes-Gavilán CG \& Salazar N. Intestinal short chain fatty acids and their link with diet and human health. Frontiers in Microbiology 20167 185. (https://doi.org/10.3389/fmicb.2016.00185)

19 Tolhurst G, Heffron H, Lam YS, Parker HE, Habib AM, Diakogiannaki E, Cameron J, Grosse J, Reimann F \& Gribble FM. Short-chain fatty acids stimulate glucagon-like peptide-1 secretion via the G-protein-coupled receptor FFAR2. Diabetes 201261 364-371. (https://doi.org/10.2337/ db11-1019)

20 Wang X, He G, Peng Y, Zhong W, Wang Y \& Zhang B. Sodium butyrate alleviates adipocyte inflammation by inhibiting NLRP3 pathway. Scientific Reports 20155 12676. (https://doi.org/10.1038/ srep12676)

21 Hill C, Guarner F, Reid G, Gibson GR, Merenstein DJ, Pot B, Morelli L, Canani RB, Flint HJ, Salminen S, et al. Expert consensus document. The International Scientific Association for Probiotics and Prebiotics consensus statement on the scope and appropriate use of the term probiotic. Nature Reviews: Gastroenterology and Hepatology 201411 506-514. (https://doi.org/10.1038/nrgastro.2014.66)

22 McFarland LV, Evans CT \& Goldstein EJC. Strain-specificity and disease-specificity of probiotic efficacy: a systematic review and metaanalysis. Frontiers in Medicine 20185 124. (https://doi.org/10.3389/ fmed.2018.00124)

23 Park KY, Kim B \& Hyun CK. Lactobacillus rhamnosus GG improves glucose tolerance through alleviating ER stress and suppressing macrophage activation in $\mathrm{db} / \mathrm{db}$ mice. Journal of Clinical Biochemistry and Nutrition 201556 240-246. (https://doi. org/10.3164/jcbn.14-116)

24 Balakumar M, Prabhu D, Sathishkumar C, Prabu P, Rokana N, Kumar R, Raghavan S, Soundarajan A, Grover S, Batish VK, et al. Improvement in glucose tolerance and insulin sensitivity by probiotic strains of Indian gut origin in high-fat diet-fed C57BL/6J mice. European Journal of Nutrition 201857 279-295. (https://doi.org/10.1007/s00394-016-1317 7)

25 Lim SM, Jeong JJ, Woo KH, Han MJ \& Kim DH. Lactobacillus sakei OK67 ameliorates high-fat diet-induced blood glucose intolerance and obesity in mice by inhibiting gut microbiota lipopolysaccharide production and inducing colon tight junction protein expression. Nutrition Research 201636 337-348. (https://doi.org/10.1016/j. nutres.2015.12.001)

26 An Y, Li Y, Wang X, Chen Z, Xu H, Wu L, Li S, Wang C, Luan W, Wang $\mathrm{X}$, et al. Cordycepin reduces weight through regulating gut microbiota in high-fat diet-induced obese rats. Lipids in Health and Disease 201817 276. (https://doi.org/10.1186/s12944-018-0910-6)

27 Pokusaeva K, Fitzgeral GF \& van Sinderen D. Carbohydrate metabolism in bifidobacteria. Genes and Nutrition 20116 285-306. (https://doi. org/10.1007/s12263-010-0206-6)

28 Vrieze A, Van Nood E, Holleman F, Salojärvi J, Kootte RS, Bartelsman JF, Dallinga-Thie GM, Ackermans MT, Serlie MJ, Oozeer R, et al. Transfer of intestinal microbiota from lean donors increases insulin sensitivity in individuals with metabolic syndrome. Gastroenterology 2012143 913.e7-916.e7. (https://doi.org/10.1053/j.gastro.2012.06.031)

Received in final form 21 September 2021

Accepted 24 September 2021

Accepted Manuscript published online 24 September 2021 https://ec.bioscientifica.com https://doi.org/10.1530/EC-21-0433 (c) 2021 The authors Published by Bioscientifica Ltd
This work is licensed under a Creative Commons Attribution-NonCommercial-NoDerivatives 4.0 Internationab ficense.ifica . com at 04/26/2023 10:05:28AM 